\title{
Diversity of ticks in the wildlife screening center of São Paulo city, Brazil
}

\author{
Thiago Fernandes Martins ${ }^{1 *}$ Liliane Milanelo ${ }^{2}$ Felipe da Silva Krawczak ${ }^{1}$ Haroldo Ryoiti Furuya ${ }^{2}$ \\ Lilian Sayuri Fitorra ${ }^{2}$ Fábio Toledo das Dores $^{2}$ Valéria da Silva Pedro ${ }^{2}$ Alicia Giollo Hippolito ${ }^{2}$ \\ Marcelo Bahia Labruna ${ }^{1}$
}

${ }^{1}$ Departamento de Medicina Veterinária Preventiva e Saúde Animal, Faculdade de Medicina Veterinária e Zootecnia, Universidade de São Paulo (USP), Av. Prof. Orlando Marques de Paiva, 87, 05508-000, São Paulo, SP, Brasil. E-mail: thiagodogo@hotmail.com. *Corresponding author.

${ }^{2}$ Departamento de Águas e Energia Elétrica, Centro de Triagem de Animais Silvestres (CETAS), Parque Ecológico do Tietê, São Paulo, SP, Brasil.

ABSTRACT: The Wildlife Screening Center (CETAS) of the Tietê Ecological Park (PET), situated at the municipality of São Paulo, receives, treats and rehabilitates wild animals that have been dislodged from their natural environment due to different reasons. This study analyzed the ixodid fauna, and the rickettsial infection in these ticks, collected on wild animals received at the PET'S CETAS. During the period from March 2003 to November 2016, 936 ticks were collected from 96 wild animals (16 bird and 18 mammal species) that were sent to CETAS. The following 12 ixodid species were identified: Amblyomma aureolatum, Amblyomma brasiliense, Amblyomma calcaratum, Amblyomma dubitatum, Amblyomma longirostre, Amblyomma ovale, Amblyomma parkeri, Amblyomma sculptum, Amblyomma varium, Haemaphysalis juxtakochi, Ixodes loricatus and Rhipicephalus microplus. From 67 tick specimens tested by the Real Time PCR for rickettsiae, none were positive. The present research records for the first time in Brazil the following association between the tick stages and hosts that have never been reported before: Amblyomma sculptum nymphs on Caprimulgus parvulus, Asio clamator, Buteo brachyurus, Coragyps atratus, Amazona aestiva and Aramus guarauna, Amblyomma dubitatum nymphs on Alouatta guariba and Sphiggurus villosus, Amblyomma aureolatum adults on Bradypus variegatus, Amblyomma longirostre larvae and nymphs on A. clamator, and nymphs on Megascops choliba and Pyroderus scutatus, besides Amblyomma parkeri nymphs on Penelope obscura and Callicebus nigrifrons, and adult on Nasua nasua.

Key words: Amblyomma, Ixodes, Haemaphysalis, Rhipicephalus.

Diversidade de carrapatos no centro de triagem de animais silvestres na cidade de São Paulo, Brasil

RESUMO: O Centro de Triagem de Animais Silvestres (CETAS) do Parque Ecológico do Tietê (PET), localizado no município de São Paulo, recebe, atende e reabilita animais silvestres que vem sendo retirados do seu ambiente natural por diversas razões. Este estudo relata a diversidade de carrapatos coletados em animais silvestres recebidos no PET, assim como a pesquisa de riquétsias nestes ectoparasitas. Durante os meses de março de 2003 a novembro de 2016, foram coletados e identificados 936 carrapatos de 96 animais silvestres (16 espécies de aves e 18 de mamíferos) atendidos no CETAS. Foram identificadas 12 espécies de ixodideos: Amblyomma aureolatum, Amblyomma brasiliense, Amblyomma calcaratum, Amblyomma dubitatum, Amblyomma longirostre, Amblyomma ovale, Amblyomma parkeri, Amblyomma sculptum, Amblyomma varium, Haemaphysalis juxtakochi, Ixodes loricatus e Rhipicephalus microplus. De 67 carrapatos testados por PCR em tempo real para riquétsias, nenhum foi positivo. O presente trabalho registra pela primeira vez no Brasil as seguintes associações dos estágios de carrapatos e hospedeiros que nunca foram relatadas anteriormente: ninfas de Amblyomma sculptum em Caprimulgus parvulus, Asio clamator, Buteo brachyurus, Coragyps atratus, Amazona aestiva e Aramus guarauna, ninfas de Amblyomma dubitatum em Alouatta guariba e Sphiggurus villosus, adultos de Amblyomma aureolatum em Bradypus variegatus, larvas e ninfa de Amblyomma longirostre em A. clamator e ninfas em Megascops choliba e Pyroderus scutatus, além de ninfas de Amblyomma parkeri em Penelope obscura e Callicebus nigrifrons e adulto em Nasua nasua.

Palavras-chave: Amblyomma, Ixodes, Haemaphysalis, Rhipicephalus.

\section{INTRODUCTION}

Ticks are arthropods of the Ixodida order and Acarina subclass, comprising ectoparasites that are highly specialized in hematophagy. Different species of mammals, birds, reptiles, and amphibians serve as hosts feeding stages of ticks, namely larvae, nymphs, and adults. Ticks are considered the second largest group of vectors of human diseases, behind only mosquitoes, and the most important vectors of pathogens to wild and domestic animals (MEDIANNIKOV \& FENOLLAR, 2014).

The Wildlife Recovery Center (CRAS) of the Tietê Ecological Park (PET) of the Water and Electric Power Department (DAEE) is a Wildlife Screening Center (CETAS) class "A", according to 
IBAMA's $169^{\text {th }}$ normative instruction from February 20th, 2008. The CETAS receives wild animals from illegal wildlife trade, from apprehensions made by the Environmental Military Police, Brazilian Institute of the Environment and Renewable Natural Resources (IBAMA), Civilian, Municipal and Federal Police inspections; those rescued by the City Hall, Fire Departments, Urban Parks, Private Companies or even those brought by common citizens. Since CETAS's opening, CRAS has received more than 72 thousand wild animals, on average birds represent $84 \%$ of the animals received; mammals $5 \%$ and reptiles $11 \%$, adding up to 520 species (MILANELO \& FITORRA, 2012).

Due to the importance of knowledge of ticks fauna and the parasite-host relation in wild animals and the pathogens transmitted by ticks that parasite the Brazilian wild fauna, the aim of the present study was to analyze the ixodid fauna biodiversity and rickettsial infection in these same ticks coming from the animals received and treated at PET's CETAS.

\section{MATERIALS AND METHODS}

A total of 96 wild animals were received by the PET during the period from March 2003 to November 2016. Animals were rescued in 28 different municipalities, including São Paulo city and nearby municipalities (Table 1 and 2). Sampled animals belonged to different age groups and genders, and were identified by the PET's veterinarians and biologists. Animals comprised 21 bird and 75 mammals specimens (Table 1 and 2).

During the treatment at the PET's Veterinarian Hospital of those animals received at the CETAS, in every inspection the ticks reported on the animals were collected and forwarded alive in $70 \%$ alcohol to the Parasitic Disease Laboratory of the Faculty of Veterinary Medicine and Animal Science of University of São Paulo (FMVZ-USP). Some engorged immature ticks were placed in B.O.D incubators for ecdysis, as detailed elsewhere (LABRUNA et al., 2002). Ticks were taxonomically identified according to current taxonomic literature (BARROS-BATTESTI et al., 2006; MARTINS et al., 2010, 2016). Voucher specimens were deposited in the tick collection "Coleção Nacional de Carrapatos Danilo Gonçalves Saraiva" (CNC) of the FMVZUSP (Table 1 and 2).

Some tick specimens $(n=67$, Table 2$)$ had their DNA extracted by the guanidine isothiocyanate method (SANGIONI et al., 2005) and tested for rickettsiae by a Taqman Real-Time PCR (qPCR) targeting the citrate synthase gene (gltA) of the genus Rickettsia, according to LABRUNA et al. (2004b). This protocol uses a pair of primers (CS5 e CS6) that amplify a 147 nucleotide fragment of the rickettsial gltA gene, combined with an internal fluorogenic probe (5' 6-FAM - BHQ-1 3') of 23 nucleotides. This protocol has shown to be sensitive to the detection of one single gene copy of spotted fever group Rickettsia spp. (LABRUNA et al., 2004b). To ensure the viability of the extracted DNA, all samples that were analyzed by the qPCR for molecular detection of rickettsiae were also tested by a conventional PCR targeting a 460-bp fragment of the tick 16S mitochondrial rDNA gene (BLACK \& PIESMAN, 1994).

\section{RESULTS AND DISCUSSION}

A total of 936 tick specimens (58 larvae, 303 nymphs, 319 males and 256 females) were collected from 96 sampled wild animals, comprising 16 bird and 18 mammal species. Overall, 936 tick specimens were collected and identified to 12 distinct ixodid species (Table 1 and 2).

No rickettsial DNA was detected in any of the 67 tick specimens that were tested by qPCR targeting the gltA gene (Table 2). These same ticks yielded visible amplicons by the PCR protocol targeting the mitochondrial $16 \mathrm{~S}$ rDNA, validating our DNA extraction protocol.

While the majority of tick-host species associations reported in this study has been previously described in the literature (BARROS \& BAGGIO, 1992; PEREIRA et al., 2000; ARZUA et al., 2005; LABRUNA et al., 2005, 2009; MARTINS et al., 2006; MARTINS et al., 2011, 2013, 2015, 2016; SARAIVA et al., 2012; LAVINA et al., 2015; TEIXEIRA et al., 2017), the present study records for the first time in Brazil, the parasitism of Amblyomma longirostre larva and nymph on Asio clamator, and nymphs on Megascops choliba and Pyroderus scutatus, in addition to a nymphal record of Amblyomma sculptum on Caprimulgus parvulus, A. clamator, Buteo brachyurus, Coragyps atratus, Amazona aestiva, and Aramus guarauna in the country.

We reported a nymph of $A$. sculptum (molted to a male in the laboratory) in Tyto furcata in the municipality of Guarulhos. While there is one previous report of $A$. sculptum (published as Amblyomma cajennense) parasitizing $T$. furcata (published as Tyto alba) at the Belo Horizonte CETAS, Minas Gerais State (ANDERY et al., 
Table 1 - Ticks collected from wild birds and mammals received and treated by the Wildlife Screening Center in São Paulo city, Brazil, from March 2003 to November 2016.

\begin{tabular}{|c|c|c|c|c|}
\hline Hosts $^{+}$ & Municipality & Dates & Ticks & Accession number \\
\hline Tyto furcata & Guarulhos & III-2003 & 1N\$ Amblyomma sculptum & CNC-797 \\
\hline Saltator similis & São Paulo & VIII-2010 & 1L Amblyomma sp. & CNC-2419 \\
\hline S. similis & Diadema & $\mathrm{X}-2015$ & $2 \mathrm{~N}$ Amblyomma longirostre & CNC-3421 \\
\hline S. similis & São Paulo & VI-2015 & 1N A. longirostre & CNC-3422 \\
\hline Celeus flavescens & Mogi das Cruzes & XI-2012 & $1 \mathrm{~N} A$. longirostre & CNC-2420 \\
\hline Caprimulgus parvulus & São Paulo & IX-2012 & 1N A. sculptum & CNC-2485 \\
\hline Megascops choliba & São Paulo & II-2014 & $1 \mathrm{~N} A$. longirostre & CNC-2736 \\
\hline Penelope obscura & São Paulo & $X-2013$ & 1N Amblyomma parkeri & CNC-2737 \\
\hline Asio clamator & São Paulo & VII-2014 & $\begin{array}{c}\text { 11L } \mathrm{L}^{\mathrm{A}} \text {. longirostre; } 9 \mathrm{~L} \\
\text { Amblyomma } \mathrm{sp} .\end{array}$ & $\mathrm{CNC}-2822$ \\
\hline A. clamator & Guarulhos & VIII-2016 & $\begin{array}{c}\text { 1N } A \text {. longirostre; } 3 \mathrm{~N} A . \\
\text { sculptum }\end{array}$ & CNC-3401 \\
\hline A. clamator (2) & São Paulo & XI-2016 & $13 \mathrm{~N}$ A. sculptum & CNC-3423 \\
\hline Buteo brachyurus & Rio Grande da Serra & IX-2016 & 5N A. sculptum & $\mathrm{CNC}-3402$ \\
\hline Cariama cristata & Caçapava & IX-2010 & $\begin{array}{l}\text { 35N A. sculptum; 5L } \\
\text { Amblyomma } \text { sp. }\end{array}$ & CNC-3424 \\
\hline Crotophaga ani & Guarulhos & VII-2016 & 2L Amblyomma sp. & CNC-3425 \\
\hline Carcara plancus & Guararema & IX-2014 & $\begin{array}{l}\text { 5N A. sculptum; 1L } \\
\text { Amblyomma } \mathrm{sp} .\end{array}$ & CNC-3426 \\
\hline Tachyphonus coronatus & Suzano & V-2015 & 3L Amblyomma sp. & $\mathrm{CNC}-3427$ \\
\hline Pyroderus scutatus & Osasco & XII-2015 & $2 \mathrm{~N}$ A. longirostre & $\mathrm{CNC}-3428$ \\
\hline Aramus guarauna & São Paulo & XI-2016 & 2N A. sculptum & CNC-3429 \\
\hline Coragyps atratus & São Paulo & VI-2013 & 1N A. sculptum & $\mathrm{CNC}-3430$ \\
\hline Amazona aestiva & São Paulo & $\mathrm{X}-2016$ & 2N A. sculptum & CNC-3431 \\
\hline Didelphis aurita & Guarulhos & IX-2005 & 1F Ixodes loricatus & CNC-921 \\
\hline D. aurita (3) & São Paulo & $\begin{array}{l}\text { IX-2005; } \\
\text { XI-2013 }\end{array}$ & $1 \mathrm{M}, 5 \mathrm{~F}$ I. loricatus & CNC-922, 923, 2734 \\
\hline D. aurita & Embu das Artes & XI-2013 & 1F I. loricatus & CNC-2433 \\
\hline D. aurita & Francisco Morato & XI-2016 & 1F I. loricatus & CNC-3432 \\
\hline Alouatta guariba & São Paulo & $\mathrm{I}-2014$ & $\begin{array}{c}\text { 1F Amblyomma aureolatum; } \\
\text { 1N A. sculptum }\end{array}$ & CNC-2738 \\
\hline A. guariba & Mairiporã & $\mathrm{I}-2014$ & $2 \mathrm{~N} A$. parkeri & CNC-2739 \\
\hline A. guariba (2) & Itapecerica da Serra & IX-2013 & $\begin{array}{c}\text { 1M, 3F A. aureolatum; } 1 \mathrm{~N} A . \\
\text { sculptum }\end{array}$ & CNC-3433 \\
\hline A. guariba (2) & São Paulo & X, XII-2015 & $\begin{array}{c}\text { 1N Amblyomma dubitatum; } \\
\text { 1M, 24N A. sculptum; 1L } \\
\text { Amblyomma sp. }\end{array}$ & CNC-3434 \\
\hline Callicebus nigrifrons & Nazaré Paulista & VII-2013 & 3N A. parkeri & CNC-3435 \\
\hline Bradypus variegatus & Bertioga & XII-2010 & 1F Amblyomma varium & CNC-1777 \\
\hline B. variegatus (2) & Mogi das Cruzes & XII-2010 & $\begin{array}{c}1 \mathrm{~F} \text { A. aureolatum } ; 1 \mathrm{M}, 2 \mathrm{~F} A . \\
\text { varium }\end{array}$ & CNC-2437 \\
\hline B. variegatus & Itapecerica da Serra & IV-2016 & $2 \mathrm{M} \mathrm{A.} \mathrm{varium}$ & CNC-3436 \\
\hline B. variegatus & Atibaia & XI-2016 & $2 \mathrm{M} \mathrm{A}$. varium & CNC-3437 \\
\hline B. variegatus & Mogi das Cruzes & VIII-2015 & $6 \mathrm{M}, 2 \mathrm{~F}$ A. varium & CNC-3438 \\
\hline Tamandua tetradactyla & Registro & VII-2016 & 1F Amblyomma calcaratum & CNC-3439 \\
\hline T. tetradactyla & Pariquera-Açu & IV-2015 & 6F A. calcaratum & CNC-3440 \\
\hline Dasypus novemcinctus & São Paulo & $\mathrm{I}-2013$ & $1 \mathrm{~F}$ A. sculptum & $\mathrm{CNC}-2424$ \\
\hline
\end{tabular}

M: male; F: female; L: larva; N: nymph.

${ }^{+}$One specimen of each animal species, except when stated in parenthesis.

$\phi$ This tick was collected as an engorged nymph, which molted to adult in the laboratory.

๘ These ticks were collected as engorged larvae, which molted to nymphs in the laboratory.

CNC: Tick collection "Coleção Nacional de Carrapatos Danilo Gonçalves Saraiva" of the Faculty of Veterinary Medicine and Animal Science of the University of São Paulo. 
Table 2 - Ticks collected from wild mammals received and treated by the Wildlife Screening Center in São Paulo city, Brazil, from March 2003 to November 2016.

\begin{tabular}{|c|c|c|c|c|}
\hline Hosts $^{+}$ & Municipality & Dates & Ticks & $\begin{array}{c}\text { Accession } \\
\text { number }\end{array}$ \\
\hline Leopardus pardalis & Salesópolis & IX-2012 & $2 \mathrm{~F}+2 \mathrm{~F}^{*}$ Amblyomma aureolatum & CNC-2421 \\
\hline Leopardus guttulus & Biritiba Mirim & III-2013 & 1F A. aureolatum & $\mathrm{CNC}-2422$ \\
\hline Eira barbara & $\begin{array}{l}\text { São Bernardo do } \\
\text { Campo }\end{array}$ & IV-2007 & $1 \mathrm{M}+2 \mathrm{M}^{*}, 1 \mathrm{~F}+3 \mathrm{~F}^{*}$ Amblyomma ovale & CNC-2425 \\
\hline Cerdocyon thous & Mogi das Cruzes & $\mathrm{X}-2012$ & $\begin{array}{c}2 \mathrm{M}+7 \mathrm{M}^{*}, 2 \mathrm{~F} \text { A. aureolatum; } \\
\text { sculptum }\end{array}$ & CNC-2426 \\
\hline C. thous & São Paulo & V-2013 & 1F A. aureolatum & CNC-2486 \\
\hline Nasua nasua (4) & São Paulo & $\begin{array}{l}\text { IX-2012; VI, X- } \\
2013\end{array}$ & $\begin{array}{c}\text { 5N Amblyomma brasiliense; } 1 \mathrm{M} \text { A. ovale } ; 1 \mathrm{~F} \\
\text { Amblyomma parkeri; } 1 \mathrm{~F}, 3 \mathrm{~N} \text { A. sculptum; } 1 \mathrm{~L} \\
\text { Amblyomma } \mathrm{sp} .\end{array}$ & $\begin{array}{l}\text { CNC-2431, } \\
2484,2735\end{array}$ \\
\hline N. nasua $(8)$ & São Paulo & $\begin{array}{l}\text { V, VIII, X-2013; } \\
\text { X-2015; VII- } \\
2016\end{array}$ & 1F, 25N A. sculptum; 14L Amblyomma sp. & CNC-3441 \\
\hline Sphiggurus villosus (3) & São Paulo & $\begin{array}{l}\text { III-2003; IX- } \\
\text { 2012; I-2014 }\end{array}$ & $\begin{array}{c}\text { 4N Amblyomma dubitatum; } 4 \mathrm{M}, 2 \mathrm{~F} \text { Amblyomma } \\
\text { longirostre; } 1 \mathrm{~L} \text { Amblyomma sp. }\end{array}$ & $\begin{array}{l}\text { CNC-761, } \\
2434,2741\end{array}$ \\
\hline S. villosus & Itaquaquecetuba & VIII-2010 & $1 \mathrm{M}$ A. longirostre & $\mathrm{CNC}-2428$ \\
\hline S. villosus & Poá & II-2011 & $1 \mathrm{~F}$ A. longirostre & CNC-2429 \\
\hline S. villosus & Caieiras & VIII-2006 & $1 \mathrm{M}$ A. longirostre & CNC-2430 \\
\hline S. villosus (3) & Mogi das Cruzes & IX-2012; I-2014 & $4 \mathrm{M}, 2 \mathrm{~F}+1 \mathrm{~F}^{*}, 1 \mathrm{~N}$ A. longirostre; $1 \mathrm{M} A$. parkeri & $\begin{array}{l}\text { CNC-2432, } \\
2740\end{array}$ \\
\hline S. villosus & São Paulo & $\mathrm{X}-2013$ & $5 \mathrm{M}, 1 \mathrm{~F}$ A. longirostre & CNC-3442 \\
\hline $\begin{array}{l}\text { Guerlinguetus } \\
\text { ingrami }\end{array}$ & São Paulo & IV-2015 & 12L Amblyomma sp. & CNC-3443 \\
\hline Tapirus terrestris (2) & São Paulo & $\begin{array}{l}\text { II-2010; VI- } \\
\quad 2012\end{array}$ & $\begin{array}{c}2 \mathrm{M}, 7 \mathrm{~F}, 1 \mathrm{~N} \text { A. sculptum; } 8 \mathrm{M}, 8 \mathrm{~F} \text { Haemaphysalis } \\
\text { juxtakochi }\end{array}$ & $\begin{array}{l}\text { CNC-2423, } \\
2742\end{array}$ \\
\hline Pecari tajacu & Sete Barras & II-2013 & $118 \mathrm{M}+15 \mathrm{M}^{*}, 33 \mathrm{~F}+10 \mathrm{~F}^{*}$ A. sculptum & $\mathrm{CNC}-2427$ \\
\hline P. tajacu (2) & São Paulo & I, II-2015 & $15 \mathrm{M}, 34 \mathrm{~F}$ A. sculptum & CNC-3444 \\
\hline $\begin{array}{l}\text { Mazama gouazoubira } \\
\text { (3) }\end{array}$ & Mogi das Cruzes & $\begin{array}{l}\text { V-2012; II, IX- } \\
2013\end{array}$ & 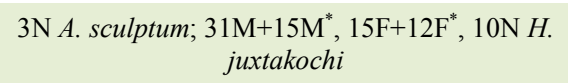 & $\begin{array}{l}\text { CNC-2436, } \\
2745\end{array}$ \\
\hline M. gouazoubira & São Paulo & XII-2013 & 2F H. juxtakochi & CNC-2743 \\
\hline M. gouazoubira & Osasco & $\mathrm{I}-2014$ & $\begin{array}{l}2 \mathrm{~N} \text { A. sculptum; } 1 \mathrm{M}, 1 \mathrm{~F} \text { H. juxtakochi; } 2 \mathrm{~F} \\
\text { Rhipicephalus microplus }\end{array}$ & CNC-2744 \\
\hline M. gouazoubira & Juquiá & V-2015 & $1 \mathrm{~N}$ A. brasiliense & CNC-3445 \\
\hline Myocastor coypus & Mogi das Cruzes & VIII-2016 & 73N A. dubitatum; 7L Amblyomma sp. & CNC-3446 \\
\hline $\begin{array}{l}\text { Hydrochoerus } \\
\text { hydrochaeris (6) }\end{array}$ & São Paulo & $\begin{array}{l}\text { VII-2005; XII- } \\
\text { 2012; VI, XI- } \\
2013\end{array}$ & $\begin{array}{l}\text { 31M, 23F, } 2 \mathrm{~N} \text { A. dubitatum; 9M, } 10 \mathrm{~F} A . \\
\text { sculptum; } 1 \mathrm{~L} \text { Amblyomma sp. }\end{array}$ & $\begin{array}{l}\text { CNC-2435, } \\
2487,2748\end{array}$ \\
\hline H. hydrochaeris & Itaquaquecetuba & $\mathrm{I}-2014$ & $14 \mathrm{M}, 11 \mathrm{~F}$ A. dubitatum $; 1 \mathrm{~F}, 1 \mathrm{~N}$ A. sculptum & $\mathrm{CNC}-2746$ \\
\hline H. hydrochaeris (2) & Guarulhos & VII, X-2013 & $6 \mathrm{M}, 5 \mathrm{~F}, 1 \mathrm{~N}$ A. dubitatum; $1 \mathrm{~F}, 5 \mathrm{~N}$ A. sculptum & CNC-2747 \\
\hline H. hydrochaeris & São Paulo & IX-2013 & $5 \mathrm{~F}, 36 \mathrm{~N}$ A. dubitatum & - \\
\hline H. hydrochaeris (2) & Cubatão & IX-2013 & $7 \mathrm{M}, 21 \mathrm{~F}, 1 \mathrm{~N}$ A. dubitatum & - \\
\hline H. hydrochaeris & Mogi das Cruzes & XI-2016 & $2 \mathrm{M}, 5 \mathrm{~F}, 6 \mathrm{~N}$ A. dubitatum; $1 \mathrm{M}, 2 \mathrm{~F}, 2 \mathrm{~N} A$. & $\mathrm{CNC}-3447$ \\
\hline
\end{tabular}

M: male; F: female; L: larva; N: nymph.

${ }^{+}$One specimen of each animal species, except when stated in parenthesis.

* Ticks tested by the PCR.

CNC: Tick collection "Coleção Nacional de Carrapatos Danilo Gonçalves Saraiva” of the Faculty of Veterinary Medicine and Animal Science of the University of São Paulo. 
2013), these authors did not specify the tick stage that was collected. Noteworthy, A. sculptum is the main vector of Rickettsia rickettsii in Brazil (MARTINS et al., 2016).

Even though Amblyomma parkeri has been stated parasitizing various species of birds and mammals in the Brazilian territory (LABRUNA et al., 2009; MARTINS et al., 2013), we demonstrated in this research for the first time the occurrence of $A$. parkeri nymphs on Penelope obscura, and Callicebus nigrifrons, and adult on Nasua nasua.

Still unprecedentedly, we reported nymphs of Amblyomma dubitatum on Sphiggurus villosus in the Brazilian territory, corroborating with previous reports that rodents are the main hosts for all stages of this tick species (LABRUNA et al., 2004a; NAVA et al., 2010). Additionally, the present study reported for the first time a nymph of A. dubitatum on Alouatta guariba.

While LABRUNA et al. (2005) reported Amblyomma aureolatum as one of the most common tick species of wild carnivores in Brazil, we reported an A. aureolatum female on Bradypus variegatus in the municipality of Mogi das Cruzes. Such unusual record has been previously reported by GUGLIELMONE et al. (2003), who recorded two $A$. aureolatum females on Bradypus spp. sloths.

This research does not reported the presence of rickettsiae in the analyzed tick specimens, it is unequivocally accepted that there has been an increase of reports of rickettsiae in ticks from wild animals during recent years in Brazil (SPOLIDORIO et al., 2012; SOARES et al., 2015; ACOSTA et al., 2016; WITTER et al., 2016). The proportion of undiagnosed diseases, especially inland, may suggest that there are even more rickettsiae associated to ticks in the national territory.

\section{CONCLUSION}

The present study contributed with important data to the ixodid fauna of wild animals in Brazil as well as on the parasite-host interface, bringing various unpublished records of ectoparasitism of ticks on wild animals in the country. These data may contribute to a more comprehensive knowledge in the biology and behavior of these arthropods in relation to their hosts in the Brazilian territory.

\section{ACKNOWLEDGMENTS}

The authors thank Alberto A. Guglielmone (INTA, Rafaela, Argentina) for providing data from his extensive archives of ticks from the Neotropical region. The present work has received financial support from the Fundação de Amparo à Pesquisa do Estado de São Paulo (FAPESP) and Conselho Nacional de Desenvolvimento Científico e Tecnológico (CNPq).

\section{REFERENCES}

ACOSTA, I.C.L. et al. Ticks (Acari: Ixodidae, Argasidae) from humans, domestic and wild animals in the state of Espírito Santo, Brazil, with notes on rickettsial infection. Veterinary Parasitology: Regional Studies and Reports, v.3, n.4, p.66-69, 2016. Available from: <http://dx.doi.org/10.1016/j.vprsr.2016.08.001>. Accessed: Nov. 15, 2016.

ANDERY, D. de A. et al. Health assessment of raptors in triage in Belo Horizonte, MG, Brazil. Revista Brasileira de Ciência Avícola, v.15, n.3, p.247-256, 2013. Available from: <http:// dx.doi.org/10.1590/S1516-635X2013000300012>. Accessed: Jan. 14, 2016.

ARZUA M. et al. Catalogue of the tick collection (Acari: Ixodidae) of the Museu de História Natural Capão da Imbuia, Curitiba, Paraná, Brazil. Revista Brasileira de Zoologia, v.22, n.3, p.623-632, 2005. Available from: <http://dx.doi.org/10.1590/ S0101-81752005000300015>. Accessed: July 17, 2016.

BARROS, D.M.; BAGGIO, D. Ectoparasites Ixodida Leach, 1817 on wild mammals in the State of Paraná, Brazil. Memórias do Instituto Oswaldo Cruz, v.87, n.2, p.291-296, 1992. Available from: <http://dx.doi.org/10.1590/S0074-02761992000200018>. Accessed: June 01, 2016.

BARROS-BATTESTI, D.M. et al. Carrapatos de importância médico-veterinária da região neotropical: um guia ilustrado para identificação de espécies. São Paulo: Vox/ICTTD-3/Butantan, 2006. 223p.

BLACK, W.C.; PIESMAN, J. Phylogeny of hard- and softtick taxa (Acari: Ixodida) based on mitochondrial 16S rDNA sequences. Proceedings of the National Academy of Sciences of the United States of America, v.91, n.21, p.10034-10038, 1994. Available from: <http://www.pnas.org/content/91/21/10034.long >. Accessed: Feb. 11, 2016.

GUGLIELMONE, A.A. et al. Amblyomma aureolatum (Pallas, 1772) and Amblyomma ovale Kock, 1844: hosts, distribution and 16S rDNA sequences. Veterinary Parasitology, v.113, n.3-4, p.273-288, 2003. Available from: <http://dx.doi.org/10.1016/ S0304-4017(03)00083-9>. Accessed: Mar. 13, 2016.

LABRUNA, M.B. et al. Ticks (Acari: Ixodidae) on wild animals from the Porto Primavera Hydroelectric power station area, Brazil. Memórias do Instituto Oswaldo Cruz, v.97, n.8, p.11331136, 2002. Available from: <http://dx.doi.org/10.1590/S007402762002000800012>. Accessed: July 11, 2016.

LABRUNA, M.B. et al. Life cycle of Amblyomma cooperi (Acari: Ixodidae) using capybaras (Hydrochaeris hydrochaeris) as hosts. Experimental and Applied Acarology, v.32, p.79-88, 2004a. Available from: $<$ http://link.springer.com/article/10.1023/ B:APPA.0000018228.05088.26>. Accessed: June 22, 2016.

LABRUNA, M.B. et al. Rickettsia species infecting Amblyomma cooperi ticks from an area in the State of São Paulo, Brazil, where Brazilian Spotted Fever is endemic. Journal of Clinical 
Microbiology, v.42, n.1, p.90-98, 2004b. Available from: <https:// www.ncbi.nlm.nih.gov/pmc/articles/PMC321730/>. Accessed: Nov. 12, 2016.

LABRUNA, M.B. et al. Ticks (Acari: Ixodida) on wild carnivores in Brazil. Experimental and Applied Acarology, v.36, n.1-2, p.149-163, 2005. Available from: <https://www.ncbi.nlm.nih.gov/ pubmed/16082932>. Accessed: Oct. 12, 2016.

LABRUNA, M.B. et al. Redescription of the female, description of the male, and several new records of Amblyomma parkeri (Acari: Ixodidae), a South American tick species. Experimental and Applied Acarology, v.49, n.3, p.243-260, 2009. Available from: $\quad<$ http://link.springer.com/article/10.1007\%2Fs10493-0099257-z>. Accessed: Dec. 25, 2015.

LAVINA, M.S. et al. Ixodids in wild animals of the mountainous plateau Region of Santa Catarina State, Brazil. Semina Ciências Agrárias, v.36, n.5, p.3173-3180, 2015. Available from: $<$ http://www.uel.br/revistas/uel/index.php/semagrarias/article/ viewFile/19119/17169>. Accessed: Dec. 25, 2015.

MARTINS, J.R. et al. First record of Amblyomma aureolatum (Pallas, 1772) (Acari: Ixodidae) parasitizing Alouatta guariba (Humboldt, 1812) (Primata: Atelidae) in Southern Brazil. Revista Brasileira de Parasitologia Veteterinária, v.15, p.203-205, 2006. Available from: <http://www.cbpv. org.br/rbpv/documentos/1542006/c154203_205.pdf $>$. Accessed: Dec. 15, 2015.

MARTINS, T.F. et al. Nymphs of the genus Amblyomma (Acari: Ixodidae) of Brazil: descriptions, redescriptions, and identification key. Ticks and Tick-borne Diseases, v.1, n.2, p.75-99, 2010. Available from: <http://dx.doi.org/10.1016/j.ttbdis.2010.03.002>. Accessed: May 26, 2016.

MARTINS, T.F. et al. Ticks on free-living wild mammals in Emas National Park, Goiás State, central Brazil. Systematic and Applied Acarology, v.16, n.3, p.201-206, 2011. Available from: $<$ http://dx.doi.org/10.11158/saa.16.3.2>. Accessed: May 26, 2016.

MARTINS, T.F. et al. Morphological description of the nymphal stage of Amblyomma geayi and new nymphal records of Amblyomma parkeri. Ticks and Tick-borne Diseases, v.4, n.3, p.181-184, 2013. Available from: <http://dx.doi.org/10.1016/j. ttbdis.2012.11.015>. Accessed: May 26, 2016.

MARTINS, T.F. et al. Ocorrência de carrapatos em animais silvestres recebidos e atendidos pelo Parque Zoológico Municipal Quinzinho de Barros, Sorocaba, São Paulo, Brasil. Brazilian Journal of Veterinary Research and Animal Science, v.52, n.4, p.319-324, 2015. Available from: <http://dx.doi.org/10.11606/ issn.1678-4456.v52i4p319-324>. Accessed: May 26, 2016.

MARTINS, T.F. et al. Geographical distribution of Amblyomma cajennense (sensu lato) ticks (Parasitiformes: Ixodidae) in Brazil, with description of the nymph of $A$. cajennense (sensu stricto). Parasites and Vectors, v.9, n.186, p.1-14, 2016. Available from: $<$ http://parasitesandvectors.biomedcentral.com/articles/10.1186/ s13071-016-1460-2>. Accessed: May 26, 2016.
MEDIANNIKOV, O.; FENOLLAR, F. Looking in ticks for human bacterial pathogens. Microbial Pathogenesis, v.77, p.142-148, 2014. Available from: <http://dx.doi.org/10.1016/j. micpath.2014.09.008>. Accessed: Nov. 02, 2016.

MILANELO, L.; FITORRA, L.S. Centro de recuperação de animais silvestres "Orlando Vilas Boas" - Parque Ecológico do Tietê (CRAS-PET-DAEE). Centros de Triagem e Áreas de Soltura de Animais Silvestres no Estado de São Paulo. São Paulo: Revista CETAS e ASMs no Estado de São Paulo Relatório de Atividades 2012, Superintendência do IBAMA no Estado de São Paulo, Ministério do Meio Ambiente, 2012. p.2327. Available from: $<$ http://www.ceo.org.br/campanhas/soltura/ Revista\%20IBAMA\%20CETAS\%20e\%20ASMS\%204\%20 -\%202012.pdf>. Accessed: Nov. 03, 2016.

NAVA, S. et al. Hosts, distribution and genetic divergence (16S rDNA) of Amblyomma dubitatum (Acari: Ixodidae). Experimental and Applied Acarology, v.51, n.4, p.335-351, 2010. Available from: $<$ http://link.springer.com/article/10.1007\% 2Fs10493-009-9331-6>. Accessed: Nov. 14, 2016.

PEREIRA, M.C. et al. Ticks (Acari: Ixodidae) associated with wild animals in the Pantanal region of Brazil. Journal of Medical Entomology, v.37, n.6, p.979-983, 2000. Available from: <http://dx.doi.org/10.1603/0022-258537.6.979>. Accessed: June 16, 2016.

SANGIONI, L.A. et al. Rickettsial infection in animals and Brazilian spotted fever endemicity. Emerging Infectious Diseases, v.11, n.2, p.265-270, 2005. Available from: <https:// www.ncbi.nlm.nih.gov/pmc/articles/PMC3320454/pdf/04-0656. pdf>. Accessed: July 01, 2016.

SARAIVA, D.G. et al. Ticks (Acari: Ixodidae) associated with small terrestrial mammals in the state of Minas Gerais, southeastern Brazil. Experimental and Applied Acarology, v.58, n.2, p.159166, 2012. Available from: <http://link.springer.com/article/10.10 07\%2Fs10493-012-9570-9>. Accessed: Jan. 19, 2016.

SOARES, H.S. et al. Ticks and rickettsial infection in the wildlife of two regions of the Brazilian Amazon. Experimental and Applied Acarology, v.61, n.1, p.125-140, 2015. Available from: <http:// link.springer.com/article/10.1007\%2Fs10493-014-9851-6>. Accessed: Jan. 14, 2016.

SPOLIDORIO, M.G. et al. Rickettsial infection in ticks collected from road-killed wild animals in Rio de Janeiro, Brazil. Journal of Medical Entomology, v.49, n.6, p.1510-1514, 2012. Available from: $<$ http://dx.doi.org/10.1603/ME12089>. Accessed: Sept. 26, 2016.

TEIXEIRA, R.H.F. et al. Ixodídeos coletados parasitando animais selvagens no Zoológico de Sorocaba, Estado de São Paulo, Brasil. Revista de Educação Continuada em Medicina Veterinária e Zootecnia do CRMV-SP, 2017.

WITTER, R. et al. Rickettsial infection in ticks (Acari: Ixodidae) of wild animals in midwestern Brazil. Ticks and Tick-borne Diseases, v.7, n.3, p.415-423, 2016. Available from: <http://dx.doi. org/10.1016/j.ttbdis.2015.12.019>. Accessed: Nov. 06, 2016. 